\title{
IL CASO ITALIA TRA BOOM MIGRATORIO E CRISI POLITICA. RIFLESSIONI SU UN PAESE MALATO.
}

\author{
ITALY'S CASE: MIGRATING BOOM AND POLITICAL CRISIS. \\ REFLECTIONS ON A SICK COUNTRY
}

\author{
Giuliano Tardivo \\ Universidad Rey Juan Carlos, Madrid. España/Spain \\ giulianotardivo@hotmail.com
}

Recibido/Received: 25/05/08

Aceptado/Accepted: 28/07/08

\section{RIASSUNTO}

Il successo, nelle elezioni italiane, di forze politiche "antisistema" come la Lega Nord o l'estrema destra, piú contrarie all'immigrazione e piú inclini a slogan intolleranti, dimostra le mille contraddizioni di una societá che ha bisogno degli immigrati e che fatica ad accettarli socialmente. La lunga transizione, incominciata nel 1992, é ben lungi dal terminare, e la crisi politica, economica, sociale, sembra aver incattivito gli italiani. Ma puó un paese con 60 milioni di oriundi perdere improvvisamente, nel giro di pochi decenni, la sua memoria storica?.Intanto, fra pregiudizi e comitati che si oppongono alla costruzione di nuove moschee, timori di invasioni e disordine legislativo, si scopre che in Italia il numero di stranieri non é poi cosí alto come molti pensano e che molti settori dell'economia continuano a richiederne di piú. In attesa di una integrazione che ancora non c'è.

\section{PAROLE CHIAVI}

Elezioni politiche, Lega Nord, Nord Italia, legge Bossi-Fini, Islam, media.

\begin{abstract}
The legislative elections in Italy witnessed the success of radical political groups extremely opposed to immigration and specially inclined to intolerant slogans, such as the Northern League (Lega Nord) and other extreme right forces; results reveal many contradictions existing in a society in need of immigrants which, at the same time, finds it difficult to accept them. The country's long transition, begun in 1992, is still far from ending, and the crisis -political, financial and social- seems to turn Italian citizens into more cruel people. Can a country with over 60 million inhabitants suddenly loose, in a short period of time, its historical memory? Meanwhile, in a context of prejudice and associations opposed to the presence of new mosques, in fear of future invasions and legislative disorder, the number of foreigners in Italy is not as high as it is thought of, and many relevant sectors in economy demand the arrival of more immigrants. The integration is longed for but not yet achived.
\end{abstract}

\section{KEYWORDS}

Political elections, Northern League, North of Italy, Bois-Fini law, Islam, mass media. 
Le elezioni politiche del 13 e 14 aprile hanno confermato, semmai ce ne fosse stato bisogno, che il sistema Italia sta attraversando, nel suo complesso, una delle crisi piú difficili e lunghe della sua, oramai piú che sessantennale, storia repubblicana.

La crisi economica, con una crescita vicina allo zero oramai da vari anni, si accompagna (e in parte forse spiega) al controverso risultato elettorale, che segnala una crescita delle forze di destra, anche xenofobe, e una riduzione, nel tessuto sociale piú ancora che nel Parlamento, preoccupante delle forze progressiste e piú aperte alla immigrazione ${ }^{1}$. Come spiegare altrimenti l'imprevisto successo della Lega Nord, che ha ottenuto un risultato sopra 1' $8 \%$, tra i migliori della sua ventennale storia, con punte del $30 \%$ in zone del Veneto e della Lombardia? O quello, piú limitato, della Destra estrema di Storace e Santaché, che procede dalle fila di Allenza Nazionale, e che non ha reciso le sue radici fasciste? Si tratta di forze antisistema, anche se presenti in forma diretta nei palazzi del potere da almeno 15 $a_{n n i}{ }^{2}$, che si richiamano a parole d'ordine grezze, primarie e che fanno breccia in un elettorato spaventato come non mai dalla crisi, dalle sfide della globalizzazione e dai mille mali della societá italiana di inzio millennio.

Come ha scritto Delumeau, un treno di paura attraversa l'Occidente e l'Italia in particolar modo e certa classe politica sembra volerne approfittare. Infatti, fra i cavalli di battaglia, e di successo, utilizzati da questi partiti, lo stop all'immigrazione selvaggia e la nuova parola d'ordine, mutuata direttamente dal linguaggio di Rudolph Giuliani, della "tolleranza zero", sono stati se non gli unici, certamente tra i principali. Perché, come diceva Durkheim a proposito del caso Dreyfus, quando una societá sta male, e la societá italiana oggi é malata come non mai, ha bisogno di trovare capri espiatori, bersagli deboli e incapaci di difendersi, a cui rinfacciare tutti i mali che patisce. Cosí prima, durante e dopo la campagna elettorale il "problema" immigrazione, in una sorta di riedizione in tempi moderni dell'antico metus punicus romano, é stato al centro dei discorsi dei politici e, di conseguenza, si é trasformato in una delle principali preoccupazioni che attraversa anche l'agenda dell'opinione pubblica. E le forze anti-immigrazione, come la Lega Nord con i suoi richiami grezzi e a volte persino volgari, e la sua idea di migranti come puri e semplici Gastarbeiter da rispedire al mittente il prima possibile, o l'estrema destra, hanno sfondato proprio laddove la presenza di migranti é piú evidente, cioé nel Nord e nelle regioni piú produttive, o nelle zone meno tutelate socialmente e culturalmente, come nelle degradate periferie urbane ${ }^{3}$. Riuscendo comunque ad aumentare sensibilmente $i$ consensi anche in zone tradizionalmente ostili e piú vicine alla sinistra, come in Emilia Romagna, dove gli analisti politici devono registrare che la monolitica subcultura rossa giá non é cosí monolitica ed inviolabile come un tempo ${ }^{4}$. La correlazione fra il dato politico uscito dalle urne e crescita notevole dei migranti anche in Emilia Romagna, oramai divenuta stabilmente la terza regione italiana per presenze straniere (dopo Lombardia e Veneto) ${ }^{5}$, é un dato su cui 
riflettere. Non é peraltro sorprendente constatare che queste forze anti-immigrazione dilagano tra i ceti popolari e subalterni o in quartieri considerati negli anni Settanta "rossi": giá nell'antica Roma, infatti, quelli che si opponenavano con piú vigore all'estensione dei diritti di cittadinanza erano proprio gli abitanti piú poveri dell'Urbs.

Tornando alla cronaca dei nostri giorni, il caso delle elezioni di Roma, dove la discussione tra i due principali candidati alla poltrona di sindaco si é giocata quasi esclusivamente su questa tematica, specie dopo l'aggressione-violenza di un rumeno contro una cittadina africana residente legalmente in Italia, é l'emblematica testimonianza dell'uso che ha fatto certa politica del tema immigrazione. Il successo della destra anche in questa cittá che ha culminato con l'entrata in Campidoglio, in qualitá di sindaco, per la prima volta nella storia repubblicana, di un leader procedente dalla destra post-fascista, non puó non essere interpretato come una richiesta di piú sicurezza, piú controlli e meno immigrazione. Una classe politica, quella dell'Italia di oggi, che dimostra anche con questi atteggiamenti, cioé soffiando sulle paure della gente o approfittando dell'emotivitá suscitata da un episodio, al contempo la sua debolezza e pochezza. In questo senso l'accento posto sulla crescita preoccupante di queste forze estreme non é fuori luogo. Infatti, cosí come accade in altri paesi europei, Spagna inclusa ${ }^{6}$, e come dimostrano vari sondaggi realizzati da istituti di ricerca, il cleavage politico destra-sinistra corrisponderebbe quasi esattamente alla predisposizione piú o meno aperturista nei confronti dei migranti. Con alcune sorprese in alcune cittá roccaforte della Lega Nord, dove il livello di integrazione sembrerebbe, secondo alcuni studi recenti promossi da un esperto di quest'area come Ilvo Diamanti ${ }^{7}$, sorprendentemente piú alto del previsto. In imprese del Nordest ${ }^{8}$ italiano dove la presenza di lavoratori dipendenti di religione musulmana é piú significativa, come nella De Longhi o nella Zanussi, giá da un po' di tempo esistono nelle mense menú speciali con carne halal ${ }^{9}$. Ma forse é un po' poco citare pochi virtuosi esempi e farli divenire emblematici. Perché si potrebberro prontamente contrapporre le prese di posizione del famigerato sindaco leghista di Treviso ${ }^{10}$, Gentilini, con le sue colorite, si fa per dire, espressioni, o le direttive fatte approvare da quello, altrettanto leghista, di Cittadella, che ha imposto un reddito minimo obbligatorio per quei migranti che vogliano prendere residenza in cittá ${ }^{11}$. O ancora la condanna eseguita dal tribunale di Verona nel 2004 contro sei esponenti in vista dello stesso movimento politico nordista, accusati di istigazione all'odio razziale contro un campo nomadi. Pertanto questa considerazione, relativa alla presunta integrazione tra migranti e autoctoni nel Nordest delle piccole imprese e dei distretti industriali, va ritenuta a mio avviso certamente vera se rapportata al tema del lavoro e dell'impiego, essendo zone a disoccupazione quasi zero e a basso tasso di criminalitá, risulta meno condivisibile invece quando si tratta di rapportarla ai rapporti umani e a tutto il resto. Le cronache e i giornali degli ultimi giorni riferiscono di un consigliere di Treviso che ha lanciato la proposta, davvero irenica, di usare metodi nazisti contro gli immigrati che delinquono: "per ogni immigrato che reca disturbo o danno, se ne puniscano dieci", 
come fecero i criminali di Hitler alle Fosse Ardeatine quando trucidarono piú di trecento civili innocenti. Rassicuranti, ma non troppo, le prese di distanza dei principali leader leghisti ${ }^{12}$. Saranno solo parole, minacce senza ripercussioni come si prestano a sottolineare vari uomini politici della stessa Lega, ma resta il fatto che, come diceva Régis Debrai, "le parole fanno politica, a volte senza saperlo" e sono comunque specchio di un certo clima.

\section{II}

Puó un paese, con 60 milioni di oriundi sparsi in giro per il mondo, improvvisamente perdere la sua memoria storica? Proprio questo dato, la recente trasformazione in paese ricettore di immigrati, insieme ai 10mila chilometri di confini, dei quali 8mila sono costituiti da coste, rende la situazione italiana particolarmente delicata, come non ha mancato di sottolineare l'ex ministro degli Interni, Enzo Bianco. L'Italia che, come dice l'editorialista Lucio Caracciolo ${ }^{13}$, ha vissuto una trasformazione copernicana da paese leader dell'emigrazione a numero due dell'immigrazione ${ }^{14}$, (secondo solo alla Spagna per numero di arrivi, non per popolazione totale), ha di fronte a sé sfide decisive. Resta un paese pieno di contraddizioni e con potenzialitá enormi. Un paese cha sta attraversando una transizione, non solo politica, che dura da almeno quindici anni e che ha perso fiducia in se stesso. Tanto che in molti denunciano, apertis verbis, che siamo tornati ad essere, come diceva in un tempo oramai lontano il buon Churchill, il ventre mollo dell'Europa. Un paese che fa fatica ad accettare lo straniero, troppe volte ancora visto come un diverso, e che al contempo sembra essere un richiamo irresistibile per molti di questi diversi. Un paese con una economia che richiede sempre piú stranieri ed una societá che fa fatica ad accoglierli. Senza contare l'assistenza agli anziani e il servizio domestico, che sono in mano praticamente in maniera esclusiva a donne provenienti da Romania, Filippine ecc., ci sono interi settori produttivi che dipendono in modo preponderante dalla forza lavoro migrante. In generale i lavori offerti ai chiamati, con una brutta espressione, lavoratori extracomunitari sono impieghi poco qualificati, nonostante la presenza di stranieri con un'istruzione superiore anche in Italia sia in aumento costante ${ }^{15}$. Tanto per citare qualche esempio le aziende che fanno raccolta di pomodori in Campania e Puglia, quella delle mele in Trentino, quelle che si dedicano alla mungitura delle vacche in Lombardia ed Emilia (ebbene sí, senza migranti forse non ci sarebbero, almeno in queste quantitá, né il parmigiano né il formaggio grana), gli hotel della Riviera Romagnola, i distretti industriali del Veneto come quello della lavorazione della pelle e delle scarpe di lusso della Riviera del Brenta, probabilmente dovrebbero chiudere bottega senza questa crescente e indispensabile presenza straniera. Resta il fatto che, come disse l'ex Presidente Prodi, c'è un'Italia che vuole gli immigrati durante il giorno e poi ne ha paura e vorrebbe cacciarli durante la notte.

Nonostante tutto l'Italia resta un paese sí con un numero significativo e crescente di immigrati, ma comunque ancora lontano dalle percentuali di altri grandi 
stati a maggior tradizione immigratoria. Tant'è che, a fronte di una opinione pubblica che percepisce, a causa della cattiva informazione, l'arrivo di maree umane di clandestini, e ha una percezione del fenomeno migratorio quantitativamente molto maggiore del dato reale, in realtá gli immigrati non sono poi cosí tanti, soprattutto in certe aree geografiche della Penisola. Secondo una recentissima indagine promossa dal Ministero degli Interni il numero di stranieri regolari presenti nello Stivale sarebbe infatti da rivedere al ribasso e si avvicinerebbe ai due milioni quattrocentomila, rendendo cosí la paventata invasione piú uno slogan politico che un dato reale ${ }^{16}$. Anche se la presenza complessiva, rapportata alla popolazione italiana, resta percentualmente piú ridotta rispetto ad altri paesi, resta la forte crescita registrata nell'arco di un quindicennio. Nel 1995, secondo fonti Istat, gli immigrati regolari erano appena 677mila. Insomma forte crescita sí, invasione di masse incontrollate no, dicono le statistiche, ma la percezione da parte dell'opinione pubblica é, purtroppo, molto differente. Un altro discorso poi andrebbe affrontato, ma in questa sede non ne abbiamo il tempo, a proposito dell'economia sommersa e del lavoro nero, cosí rilevanti in Italia, che richiede lavoratori molto spesso indocumentati da sfruttare, sottopagare ecc.

\section{III}

La politica italiana, nel suo complesso, forze di sinistra incluse, ha spesso favorito l'entrata di migranti ma non la loro integrazione, mancando un progetto complessivo che vada aldilá dell'idea degli stranieri come mera forza lavoro. Questi tre decenni di immigrazioni sono stati caratterizzati, infatti, da una totale mancanza di una politica per l'immigrazione, se si eccettuano le frequenti sanatorie degli irregolari che si succedono con scadenza periodica, a partire dal $1986^{17}$. Addirittura secondo un analista come Fabio Perocco quel poco che si é fatto é sempre stato ispirato alla negazione dei diritti piú che al loro riconoscimento. Vi é chi arriva a sostenere che, con la legge Bossi-Fini del 2002, che ha modificato in senso ancor piú restrittivo la precedente legge approvata dal centro-sinistra nel 1998 e conosciuta ai piú come legge Turco-Napolitano ${ }^{18}$, si é inaugurata una sorta di via italiana all'apartheid ${ }^{19}$. Un attacco durissimo "ai valori della equitá, della solidarietá, della democrazia", rincara la dose un esperto in materia giuridica come Livio Pepino ${ }^{20}$. Tra le misure particolarmente criticate di suddetta legge, che porta non a caso come primi firmatari i nomi dei segretari dei due partiti piú a destra nell'arco costituzionale $^{21}$, l'introduzione del reato di immigrazione clandestina per chi cerca di tornare in territorio italiano dopo una prima espulsione, le maggiori difficoltá introdotte per il ricongiungimento familiare (il che va in controtendenza rispetto alla necessitá di integrare) e il prolungamento a 60 giorni della permanenza nei criticati C.P.T., centri di permanenza temporanea, degli immigrati clandestini, in attesa di certificarne provenienza e identitá. Inoltre la stessa legge ha portato all'eliminazione della figura dello sponsor, introdotta dalla precedente misura legislativa TurcoNapolitano, che aveva ricevuto il plauso di associazioni come la Caritas, oltre a 
dimostrarsi effettiva sul piano pratico, consentendo a un cittadino italiano di farsi garante dell'ingresso di un immigrato e della successiva introduzione nel mercato del lavoro. Inoltre la nuova normativa ha ridotto i tempi consentiti a un immigrante che perde improvvisamente il lavoro per trovarne un altro prima di passare alla condizione giuridica di irregolare: da due anni a sei mesi.

Nonostante lo strepitare di questi mesi, molti dimenticano che la legge attualmente ancora in vigore che regolamenta queste tematiche é propio la legge Bossi-Fini, una legge che a detta di tutti, nonostante le pretese degli autori, ha tutto meno che risolto il problema di una adeguata politica migratoria in Italia. In realtá il sodalizio tra i due autori della legge del 2002, Bossi e Fini, rispettivamente leader della Lega Nord e di Alleanza Nazionale, sul tema dell'immigrazione si é rotto proprio durante la recentissima campagna elettorale, quando Fini, come giá fatto in passato, é tornato a ventilare l'ipotesi di estendere il voto agli immigrati regolari residenti da piú di cinque anni in Italia, perlomeno nelle tornate amministrative. $\mathrm{La}$ proposta ha ricevuto il diniego totale e assoluto di Bossi che é arrivato a minacciare la costituzione del futuro governo 22 . Parafrasando il grande Aristotele, "maestro di color che sanno" 23 , per certa politica italiana gli immigrati, come gli schiavi dell'antica Grecia, sembrano essere "sí e no uomini".

L'Italia resta un paese diviso economicamente e geograficamente in due e che non a caso vede un' alta concentrazione geografica dei migranti nelle tre o quattro regioni piú ricche del Nord industriale, che accolgono (si fa per dire, visti i problemi di integrazione) intorno al $64 \%$ degli immigrati totali che vivono in Italia. Tutto questo a fronte di un Sud cenerentola, dove risiede una quota minima del totale degli immigrati. E poi c'è un Italia che non accetta i migranti e che al contempo non presenta soluzioni perché non si rende disposta a sacrifici, come l'innalzamento dell'etá pensionabile o gli investimenti nelle aree depresse del profondo Sud (Calabria, Molise, Campania). Quest'Italia egoista non offre prospettive. Se a tutto ció aggiungiamo che le uniche politiche positive, di integrazione, le promueve la Caritas e l'associazionismo cattolico, a fronte di una totale assenza dello Stato e degli organi preposti, il quadro é presto fatto. Anche da questo punto di vista, peraltro, non mancano le contraddizioni: se c'è una Famiglia Cristiana o associazioni come l'appena citata Caritas che sono in prima fila a fianco dei piú deboli, dall'altra parte c'è un cardinale Biffi, che con la famigerata pastorale del 13 settembre 2000, invita ad aprire le porte ai soli cattolici.

Nel frattempo monta sempre piú l'ondata xenofoba, alimentata anche dal linguaggio di certa classe politica nazionale e locale, e i fatti di cronaca nera che hanno alla radice motivi razziali si fanno quotidiani: ogni venticinque ore c'é un immigrante che denuncia un'aggressione razzista. L'Italia del 2008 sembra presentarsi innanzi alle sfide della globalizzazione con le parole antimoderne di un cristiano come Ferdinando I rivolto agli invasori musulmani: "Emigrate alle vostre terre, piú in lá dello Stretto, e lasciateci il nostro paese!". 
Tornando ad un'analisi piú dettagliata del fenomeno, tra le comunita piú numerose e che hanno piú problemi ad integrarsi, risalta quella nord africana, seconda solo a quella rumeno-albanese (quest'ultima peró negli ultimi anni sta crescendo molto di piú rispetto a quella marocchina e tunisina ${ }^{24}$ ). Si tratta anche della comunitá che suscita piú pregiudizi e risentimenti: solo il $34 \%$ degli italiani, secondo un recente studio, ha una opinione positiva delle persone di religione islamica residenti in Italia, a fronte di un $55 \%$ che individua proprio tra gli immigrati di questa religione coloro che piú problemi sociali generano. Addirittura un italiano su tre si opporebbe, secondo una recentissima indagine, alla costruzione di nuove moschee e luoghi di culto ${ }^{25}$. Un razzismo popolare che trova avallo, se vogliamo, in una sorta di razzismo istituzionale, che sfocia nel non riconoscimento giuridico della religione islamica in Italia, al contrario di ció che succede con altre confessioni. Non é un fenomeno di poco conto, se si pensa che l'Islam é ormai, a tutti gli effetti, una presenza duratura nel nostro territorio e costituisce, in termini numerici, la seconda religione del paese. Ma, come dice con ragione un sociologo del calibro di Stefano Allievi, molti di quelli che parlano preoccupati di immigrazione e Islam poi, se vai a vedere, non sono mai entrati físicamente dentro una moschea ${ }^{26}$. In piú gli stessi mezzi di comunicazione sono soliti tratteggiare l'Islam come se fosse un monolito, un blocco costituito da terroristi pronti ad immolarsi per Allah ${ }^{27}$. In realtá, come ben dice lo scrittore algerino Abdel Malik, che conosce da vicino per esempio la comunitá islamica di Milano, tra l'islam della moschea milanese di via Padova e l'islam che si predica in via Janner c'è un mare di differenze. L'idea dell'Islam come realtá plurale é un'altra realtá che non viene trasmessa dai nostri media ed agenti culturali $^{28}$. Non esiste un unico Islam: come direbbero le tribu di Java "in campi differenti si incontrano cavallette anch'esse differenti, una diversa dall'altra". Proprio la mancanza di conoscenza, e la diffidenza é la madre di tutti i razzismi. Perché, al contrario di ció che pensava Weber, gli uomini possono anche avere religioni e credenze differenti, ma i corpi e gli stomaci ${ }^{29}$ alla fin fine sono gli stessi.

Anche una parte della memoria storica, come spiegato anteriormente, puó rappresentare un punto di contatto e di incontro fra stranieri ed autoctoni. Se il fenomeno migratorio va affrontato obbligatoriamente con una prospettiva multidisciplinare, va certamente sottolineato, a mio avviso, che per le migrazioni nel loro complesso e per il caso italiano, che stiamo pur brevemente trattando di descrivere, a maggior ragione, restano dei punti di partenza imprescindibili le riflessioni di sociologi illuminati, come, uno su tutti, Alfred Schütz, o Norbert Elias. La mitificazione della societá italiana prima del boom del fenomeno migratorio, cioé antecedente alla metá degli Ottanta, che molti italiani, laudatores temporis acti, ingiustamente celebrano, addebitando agli altri da noi le cause di tutti i mali, ricorda da vicino le analisi di Elias sugli abitanti di Winston Parva ${ }^{30}$ da lui studiati, che mitificavano il passato per difendersi dai nuovi arrivati ${ }^{31}$. 
Tutto ció, va aggiunto, diventa ancor piú grave, se si prende in considerazione la storia italiana, che ben giustifica la definizione data da Claude Levi Strauss dell'Islam come l'Oriente d'Occidente ${ }^{32}$. Non c'è quell'alteritá assoluta e totale che molti immaginano fra mondo cristiano e Islam, e questo discorso vale in modo particolare per l'Italia. La presenza araba nell'Italia del Sud e specialmente in Sicilia, non confrontabile all'esperienza di Al Andalus durata praticamente otto secoli, é comunque significativa e durante due secoli ha contribuito a forgiare caratteri, costumi, abitudini. Questo é un dato obiettivo che molto spesso, a torto, si dimentica con troppa facilitá. Se la convivenza armoniosa fu possibile nella Sicilia del secolo $X$ d.C. a maggior ragione dovrebbe essere non solo possibile, ma addirittura naturale, in tutta Italia circa un millennio dopo. É triste ammetterlo, ma per certi versi nella Sicilia araba del secolo X e XI c'erano piú integrazione e libertá che nell'Italia di oggi. Valga come esempio che la Palermo delle trecento moschee, la antica Balarm, capitale del regno arabo in Sicilia, oggi ha una sola moschea riconosciuta, nonostante il numero di tunisini e marocchini non sia insignificante. Mentre in altri territori dove la presenza islamica é piú forte, come in alcune cittá del Nord, e gli unici punti di ritrovo per pregare sono centri fai da te, ricavati in garage e scantinati, fa specie notare come la popolazione locale, alimentata dalla Lega Nord e da altri partiti, si sia in alcuni casi opposta alla costruzione di moschee in piena regola. Ma senza moschee e senza rispetto dell'altro, non avremo mai una piena integrazione. Nel 1995, tanto per fare un esempio fra i tanti possibili, un gruppo di cattolici integralisti, guidati dall'ex Presidente della Camera Irene Pivetti, celebrarono una messa riparatrice dopo l'inaugurazione della Grande Moschea di Roma. Queste reazioni, come direbbe Richard Buillet, sono del tutto equiparabili ai dettami ultrareligiosi di certo fondamentalismo islamico e servono a fare contenti i mille Huntington o predicatori di sventure sparsi a bizzeffe in giro per il mondo. Solo il riconoscimento e il rispetto delle differenze e al contempo una maggiore empatia, l'Einfühlung di husserliana memoria, con chi viene da paesi, tradizioni e religioni differenti, potrá far superare le attuali difficoltá di convivenza.

\section{V}

Anche i media con la ricerca continua di sensazionalismo giocano un ruolo di primo piano, alimentando fobie e pregiudizi. Sono loro i responsabili principali dell'equazione, oramai consolidata agli occhi del grande pubblico, fra immigrazione, criminalitá, bisogno di sicurezza ecc. Perché, come dice una esperta della questione come Ribka Sibhatu parlano dell'immigrazione presentandone solo le negativitá. Negli studi televisivi effettivamente si chiamano a discutere di immigrazione politici e questori, personaggi bizzarri e leghisti scatenati, ma mai lo straccio di un migrante che racconti la sua esperienza di vita, di sofferenza, di esclusione, se si esclude un articolo-inchiesta pubblicato qualche anno fa da Repubblica, intitolato "Gli stranieri ci giudicano: siete succubi delle donne"33. Insomma, un po' pochino, oserei dire. Si presenta a volte anche con ragione il problema della sicurezza, sottolineando con 
troppa enfasi che degli oltre 53mila detenuti che sovraffollano le carceri italiani ben 15 mila sono extracomunitari, e la maggior parte di questi irregolari o clandestini. Peró non si presentano, con altrettanto vigore, quei casi di migranti che ce l'hanno fatta, che hanno fatto intrapresa, hanno, tra mille difficoltá, vinto le barriere dell'intolleranza o della povertá. E soprattutto si parla in modo generico di albanesi, criminali romeni ecc. Dimenticandosi che in uno stato di diritto, come si presume sia ancora l'Italia, la responsabilitá penale é individuale. I telegiornali e giornali piú vicini al centro-destra, come TG5, TG4, TG1, sembrano quelli piú inclini a questa lettura e a questa ermeneutica negativa del fenomeno migratorio. Mentre Repubblica, Il Corriere della Sera e il TG3, dall'altra parte, parlano talora anche della necessitá per l'economia italiana di forza migratoria, della crescita demografica che dipende quasi per intero dagli stranieri e di altri aspetti positivi correlazionati con la tematica migratoria. Ribka Sibhatu, che ha seguito durante un mese i principali media e il loro approccio alle tematiche migratorie, ricorda che il TG5 non ha avuto pietá nemmeno di fronte al caso di una piccola bimba di 4 anni, vittima di uno spiacevole caso di violenza, il cui padre di religione islamica aveva chiesto l'applicazione della sharia per il suo brutale violentatore. É un sentimento davvero cosí diverso a quello che spinge certi cattolici a richiedere la reintroduzione della pena di morte, quando subiscono disgrazie simili?

Ma il tema dell'immigrazione, con le relative polemiche sulla sicurezza ${ }^{34}$, é solo il riflesso, forse piú evidente, di un paese impantanato in una profonda crisi morale, politica, economica, iniziato, come minimo, ad inizio anni Novanta. Sono passati sedici anni dall'annus horribilis, 1992, quando le inchiesta di Tangentopoli facevano cadere il vecchio sistema di potere, la Lira si vedeva obbligata a uscire dallo Sme, il presidente del Consiglio Amato varava una finanziaria di lacrime e sangue, Craxi faceva l'appello ai correi ${ }^{35}$ e si registrava il primo trionfo della Lega Nord $^{36}$. Nonostante il lungo periodo di tempo alle spalle la lunga transizione italiana é ben lungi dal terminare e le divisioni e i problemi, dopo un primo entusiasmo inziale, restano tutti intatti. Basti pensare che neanche una data come il 25 aprile, festa che ricorda la liberazione dal nazi-fascismo e la fine della guerra, é un valore universale che accomuna tutti gli italiani, come dimostrano le polemiche di questi giorni. Forse, come diceva Wilson per la sua America, l'Italia "apparirá in piena luce solo quando tutti sapranno che essa pone i diritti umani sopra ogni altro diritto e che la sua bandiera non é solo sua ma dell'umanitá intera".

\section{NOTAS}

${ }^{1}$ La sinistra radicale, raccolta nel cartello elettorale chiamato Sinistra arcobaleno, per la prima volte in 63 anni di Repubblica non avrá rappresentanti in Parlamento.

${ }^{2}$ Storace é stato per 4 anni presidente della regione Lazio e Bossi, presidente della Lega Nord, a lungo ministro di un governo, quello di Berlusconi, che ha peraltro prodotto nel 2002 la piú grande regolarizzazione di stranieri irregolari della storia (646mila regolarizzazioni).

${ }^{3}$ Piú del $60 \%$ degli immigrati residenti in Italia risiede nelle regioni del Nord. 


\section{Giuliano Tardivo}

${ }^{4}$ ALFIERI, M. "Immigrati e fisco, l'Emilia svolta", il Sole 24 ore, 16 aprile 2008, p.4. I politologi italiani sono soliti distinguere fra una subcultura rossa, dominante in Emilia e Toscana, e una subcultura bianca per il Veneto, democristiano prima e leghista-berlusconiano ora.

${ }^{5}$ Vedi Osservatorio Regionale sull'Immigrazione, Immigrazione straniera in Veneto, Milano, Franco Angeli, 2007.

${ }^{6}$ Come si evince dalla lettura di CAZORLA, J. "La frontera Sur de Europa: motivaciones y consecuencias sociopolíticas de la migración", Reis, 109, pp. 249-50, anche in Spagna il maggior rifiuto verso i migranti si registra tra le persone anziane, con basso livello di studio e piú radicali da un punto di vista politico e religioso.

DIAMANTI, I. E. y PORCELLATO, N. "Sorpresa nel Nordest l'integrazione funziona", Limes, 4 2007, pp. 47-62.

${ }^{8}$ Il Nordest é un'area geografiche, con forti connotazioni politiche ed economiche, che comprende il Veneto, il Friuli e il Trentino. Per saperne di piú un primo approccio interessante lo fornisce il libro del giornalista Gian Antonio Stella Schei edito da Mondadori.

${ }^{9}$ Vedi ALLIEVI, S. (2003): Islam italiano. Viaggio nella seconda religione del paese, Torino, Einaudi, p. 159.

${ }^{10}$ Treviso nonostante le intemperanze del sindaco é la quinta cittá italiana con il piú alto numero di stranieri residenti, dopo Milano, Roma, Brescia e Torino e prima di Vicenza, altra cittá del "mitico" Nordest delle piccole imprese.

${ }^{11}$ Vedi FUMAGALLI, M. "Senza reddito, niente diritto di residenza", Il Corriere della Sera, 18 novembre 2007 , p. 9.

12 Vedi FUMAGALLI, M. "Immigrati, servono metodi SS", Il Corriere della Sera, 5 dicembre 2007, p. 27.

${ }^{13}$ CARACCIOLO, L. "Le vite degli altri", Limes 4, 2007, p.9.

${ }^{14}$ Se negli Settanta gli stranieri residenti in Italia erano appena 300mila, inclusi i comunitari, a inizio 2006 se ne contavano quasi dieci volte di piú. Vivono infatti tra i due milioni quattrocentomila, secondo l'Osservatorio sociale per l'Immigrazione, e i 3 milioni e passa, secondo la Caritas, di immigrati in territorio italiano. La disparitá dei dati dimostra la difficoltá di fare calcoli precisi ed attendibili. Inutile aggiungere che il conteggio diventa quasi impossibile quando si tratta di contare il numero di clandestini.

15 Vedi a questo propósito REYNIERI, E. "De la economía sumergida a la devaluación profesional: nivel educativo e inserción en el mercado de trabajo de los inmigrantes en Italia", Reis, 116 oct.-dic. 2006, pp. 213-237.

${ }^{16}$ Vedi CUSTODERO, A. "Un italiano su tre contro le moschee", La Repubblica, 30 aprile 2008 , p. 20.

${ }^{17}$ Molto importanti da un punto di vista quantitativo sono state le sanatorie del 1990, con 217 mila regolarizzazioni, quella del 1999 con altre 250mila regolarizzazioni. Fino alla giá citata sanatoria del 2002, con piú di 600mila regolarizzazioni, promossa dal duo Bossi-Fini.

${ }^{18} \mathrm{Su}$ questa legge e sulla legislazione italiana a proposito di immigrazione fino al 2001 vedi l'interessante studio di SOLANES CORELLA, A. (2001): El espejo italiano. Un estudio de la normativa sobre la inmigración en Italia, Madrid, Dykinson, 2001.

${ }^{19}$ PEROCCO, F., "L'apartheid italiano", BASSO, P. e PEROCCO, F. (2003): Gli immigrati in Europa, Milano, Franco Angeli, p. 221.

20 PEPINO, L. "La legge Bossi-Fini. Appunti su immigrazione e democrazia", www.francoangeli.it

${ }^{21}$ In realtá risulta difficile definire secondo gli schemi tradizionali del continuum destrasinistra un partito regionalista con tendenze secessioniste come la Lega. Perché, come ha detto lo stesso professor Sartori, la Lega prende voti anche fra ex elettori di sinistra, per cui é 
difficilmente inquadrabile dentro i vecchi schemi ideologici del '900. Secondo una politologa come Simona Colarizi l'attibuto anti-ideologico e il pragmatismo sarebbero le caratteristiche fondamentali della Lega della prima ora. Resta a mio avviso, almeno sulle tematiche migratorie, un partito decisamente orientato verso posizioni estreme, lontane dal moderatismo conservatore del Partito Popolare europeo, tanto per fare un esempio.

22 OREFICE, P. "Voto agli immigrati, é scontro tra Bossi e Fini", Il Messaggero, 1 aprile 2008 , p.9.

${ }^{23}$ Cosí Dante definisce il grande filosofo da lui incontrato nel viaggio infernale nel canto IV della Divina Commedia.

${ }^{24}$ Come si puó leggere in REA, A. "La europeización de la política migratoria y la trasformación de la otredad", Reis, 116, oct.-dic. 2006, pp. 157-183, non solo in Italia, ma in tutta l'Europa del Sud, la manodopera proveniente dall'Est Europeo sta progresivamente sostituendo quella di procedenza marocchina.

${ }^{25}$ ANGELI, F. "L'Islam fa paura: un italiano su tre non vuole moschee", Il Giornale, 30 aprile 2008, p. 17.

${ }^{26}$ Vedi ALLIEVI, S., o.c., p. 8.

${ }^{27}$ Addirittura secondo un recente sondaggio il 17\% degli italiani dichiara di diffidere degli islamici perché potenziali terroristi. Vedi ANGELI, F., o.c., p. 17.

${ }^{28}$ SIBHATU, R. (2004): Il cittadino che non c'è. L'immigrazione nei media italiani, Roma, Edup, p. 71.

${ }^{29}$ Celebre la sua frase a proposito dei contadini polacchi, dotati, a suo dire, di stomaci e corpi differenti.

${ }^{30} \mathrm{Si}$ tratta di un quartiere operaio, vicino a Leicester, che ricevette numerosi lavoratori da fuori e che venne a lungo studiato dal grande sociologo.

${ }^{31}$ PERRONE, L. (2005): Da straniero a clandestino, Napoli, Liguori Editori, p. 122.

${ }^{32}$ Vedi FIERRO, M. “¿Se puede hablar de una civilización islamo-cristiana?”, Revista de Libros, 123 marzo 2007, pp. 3-5.

${ }^{33} \mathrm{Si}$ tratta di un articolo pubblicato su Repubblica nell'oramai lontano 14 dicembre 2000.

34 Secondo uno studio realizzato da Demos-Coop il 43\% del campione rappresentativo relaziona gli immigrati con il problema della sicurezza. Vedi BORDIGNOS, F. e CECCARINI, L., "Gli altri tra noi”, Limes, 4 2007, pp. 35-45.

${ }^{35}$ Si tratta del famoso discorso pronunciato da Craxi, segretario del P.S.I., che accusó tutti i partiti dell'arco costituzionale, senza eccezioni, di essere corresponsabili del sistema del malaffare e delle tangenti che finanziava la politica italiana.

${ }^{36}$ Per saperne di piú vedi COLARIZI, S. Storia dei partiti nell'Italia repubblicana, RomaBari, Laterza, 1996.

\section{BIBLIOGRAFÍA}

ALFIERI, M. (2008): “Immigrati e fisco, l'Emilia svolta”, il Sole 24 ore, 16 aprile: 4.

ALLIEVI, S. (2003): Islam italiano. Viaggio nella seconda religione del paese, Torino, Einaudi.

ANGELI, F. (2008): “L'Islam fa paura: un italiano su tre non vuole moschee”, Il Giornale, 30 aprile: 17.

BASSO, P. e PEROCCO, F. (2003): Gli immigrati in Europa, Milano, Franco Angeli.

BOLAFFI, G. (2001): I confini del patto, Torino, Einaudi.

BORDIGNOS, F. e CECCARINI, L. (2007): "Gli altri tra noi", Limes, 4: 35-45.

CAMPO, G. (2007): “Cittadella e dintorni”, Diritto-immigrazione-cittadinanza, 4: 63-70. 
CARACCIOLO, L. (2007): "Le vite degli altri”, Limes, 4: 9.

CARITAS-MIGRANTES (1998-2007): Immigrazione. Dossier statistico 1998-2007, Roma, Caritas.

CAZORLA, J. (2006): "La frontera Sur de Europa: motivaciones y consecuencias sociopolíticas de la migración”, Reis, 109: 249.

COLARIZI, S. (1996): Storia dei partiti nell'Italia repubblicana, Roma-Bari, Laterza.

CUSTODERO, A. (2008): "Un italiano su tre contro le moschee", La Repubblica, 30 aprile: 20.

DIAMANTI, I. e PORCELLATO, N. (2007): “Sorpresa nel Nordest l'integrazione funziona”, Limes, 4: 47-62.

FIERRO, M. (2007): “¿Se puede hablar de una civilización islamo-cristiana?”, Revista de Libros, 123: 3-5.

FONDAZIONE ISMU (1998-2007): Rapporto sulle migrazioni in Italia 1998-2007, Milano, Franco Angeli.

FUMAGALLI, M. (2007a): "Immigrati, servono metodi SS", Il Corriere della Sera, 5 dicembre: 27.

-(2007b) "Senza reddito, niente diritto di residenza", Il Corriere della Sera, 18 novembre: 9.

OREFICE, P. (2008): "Voto agli immigrati, é scontro tra Bossi e Fini”, Il Messaggero, 1 aprile: 9.

OSSERVATORIO REGIONALE SULL'IMMIGRAZIONE (2007): Immigrazione straniera in Veneto, Milano, Franco Angeli.

PEPINO, L., "La legge Bossi-Fini. Appunti su immigrazione e democrazia", www.francoangeli.it

PERRONE, L. (2005): Da straniero a clandestino, Napoli, Liguori Editori.

REA, A. (2006): "La europeización de la política migratoria y la trasformación de la otredad", Reis, 116, oct.-dic.: 157-183.

REYNIERI, E. (2006): "De la economía sumergida a la devaluación profesional: nivel educativo e inserción en el mercado de trabajo de los inmigrantes en Italia", Reis, 116 oct.dic.: 213-237.

SIBHATU, R. (2004): Il cittadino che non c'è. L'immigrazione nei media italiani, Roma, Edup.

SOLANES CORELLA, A. (2001): El espejo italiano. Un estudio de la normativa sobre la inmigración en Italia, Madrid, Dykinson.

\section{Breve currículo:}

\section{Giuliano Tardivo}

Investigador de la Universidad Rey Juan Carlos. Licenciado en Ciencias Políticas y Sociales por la Universidad de Padua (Italia). Máster en "Integración Europea" (Univ. de Padua) y otro en "Gestión e Investigación de la Comunicación empresarial" (URJC). Finalizado un doctorado en "Comunicación cultural e identidad entre Iberoamérica y Europa" (URJC). Realizó su tesina en Italia con el título Felipe González y Bettino Craxi: dos socialismos al espejo y actualmente está trabajando en una tesis doctoral sobre "Italia y España, dos países cara a cara. Desde Al Ándalus y Siqilliya hasta el actual fenómeno migratorio. Panorámica sobre las similitudes y las diferencias entre dos de los principales países receptores de migrantes musulmanes en Europa”. 